\title{
Anatomical considerations on the place and formation of the renal veins
}

Discipline of anatomy, Department I - preclinical disciplines, Faculty of medicine, University "Ovidius" Constanţa

\begin{abstract}
Our study on the venous vasculature of the kidney (the location and the formation of the renal veins) was performed on a total of 148 cases, using as working methods the dissection, the injection of contrast medium followed by radiography and the plastic injection followed by corrosion. Not all anatomical landmarks could be studied in all cases, each anatomical landmark being assessed on a number of different cases. The left renal vein formation was studied on 124 cases and we have found that the left renal vein may appear inside the renal sinus (intrarenal or intrasinusal) in $13.71 \%$ of cases; in $17.74 \%$ of the cases the confluence of the tributaries of the renal was at the medial border of the kidney, so juxtarenal and the most common form was the extrarenal vein $(68.55 \%$ of cases). The level of appearance of the right renal vein was studied on 122 cases, describing the following patterns: intrasinusal in $19.67 \%$ of cases, juxtarenal in $27.87 \%$ of cases and extrarenal in $52.46 \%$ of cases.

The formation of renal veins was studied on a number of 114 cases, finding the following variations: in 56.14 $\%$ of the cases the renal vein formed from two venous trunks, in $38.60 \%$ of cases from three venous trunks and in $5.26 \%$ of cases from four venous trunks; we did not found cases of renal vein formed from more than 4 tributaries.
\end{abstract}

Keywords: renal vessels - place and formation

\section{Iliescu D.M.}

Department of Anatomy, Faculty of medicine, University "Ovidius" Constanţa, Romania

Aleea Universitatii, Nr. 1, Campus B,

Constanţa, Romania

dan@anatomie.ro

\section{Introduction}

The renal veins provide the venous circulation of the kidneys, of the perirenal adipose capsule, renal pelvis, upper ureter and, for the left renal vein, a significant part of the left suprarenal gland. Usually two (it may also appear double renal veins), they are large, the most voluminous after the hepatic veins, among the inferior vena cava tributaries [1].

The renal veins are formed, most commonly, outside the hilum, rarely at this level, by the union of three to five main tributaries from the sinus pericalyceal arches. A late renal vein formation is much less common than the early division of the artery, so its relations with the arterial branches are very variable [Hovelacque, quoted by 2]. The renal vein trunk is formed mostly by the anterior venous system or prepielic, consisting of two very large venous branches, one superior and one inferior; sometimes three or more prepielic branches may occur [2]. The posterior venous system (retropyelic) is smaller and can be considered as a mere tributary of the renal vein trunk. After the formation, the renal vein is oriented transversally, lateral- medial and slightly infero-superior, ending on the corresponding side of the inferior vena cava at the level of the lower border of the first lumbar vertebra or intervertebral disc L1-L2 [1,2]. The left renal vein has a very oblique trajectory than the right one; the inferior vena cava end is almost at right angles on the right and 
acute angle on the left. The renal veins rarely end at the same level, most often the end of the left renal vein being higher than the right one $[1,2,3,4,5]$. The gap between the ending levels of the renal veins is at least $10 \mathrm{~mm}$ and maximum $15 \mathrm{~mm}[5,6,7]$. These differences appear due to the different embryological development [8]. The left renal vein, very variable in direction, is more horizontal than the right one, crosses the anterior face of the aorta, in the angle formed with the superior mesenteric artery, between the origin of the superior mesenteric artery and the origin of the gonadal arteries; the aorta crossing is made, generally, at the level of the second lumbar vertebra $[2,3,4,5]$.

\section{Material and methods}

Our study on the venous vasculature of the kidney was performed on a total of 148 cases, represented by the eviscerated kidneys (fresh and formalin preserved) or in situ, in the halls of in anatomy lab and fresh renal organic blocks: kidney with perirenal fat and the corresponding segments of the abdominal aorta and inferior vena cava. We used as methods the dissection, the injection of contrast medium followed by radiography and the plastic injection followed by corrosion. Not all anatomical landmarks could be studied in all cases, each anatomical landmark being assessed on a number of different cases.

\section{Results and discussions}

The level of formation of the left renal vein was studied on 124 cases. We have found that the left renal vein may form inside the renal sinus in 17 cases ( $13.71 \%$ of cases), intrarenal or intrasinusal, thus it exits into the hilum of the kidney as a single trunk. In 22 cases the confluence of the tributaries is at the level of the medial border of the kidney, so juxtarenal (17.74\% of cases).

The most common pattern is the extrarenal formation of the renal vein, in 85 cases $(68.55 \%$ of cases), that we described several types:

- closer to the medial border of the kidney, in 30 cases $(24.20 \%$ of cases);

- mid distance aorta-kidney, in 39 cases (31.45\% of cases);

- on the lateral side of the aorta in 11 cases (8.87\% of cases);

- on the antero-lateral side of the aorta in 5 cases $(4.03 \%$ of cases).

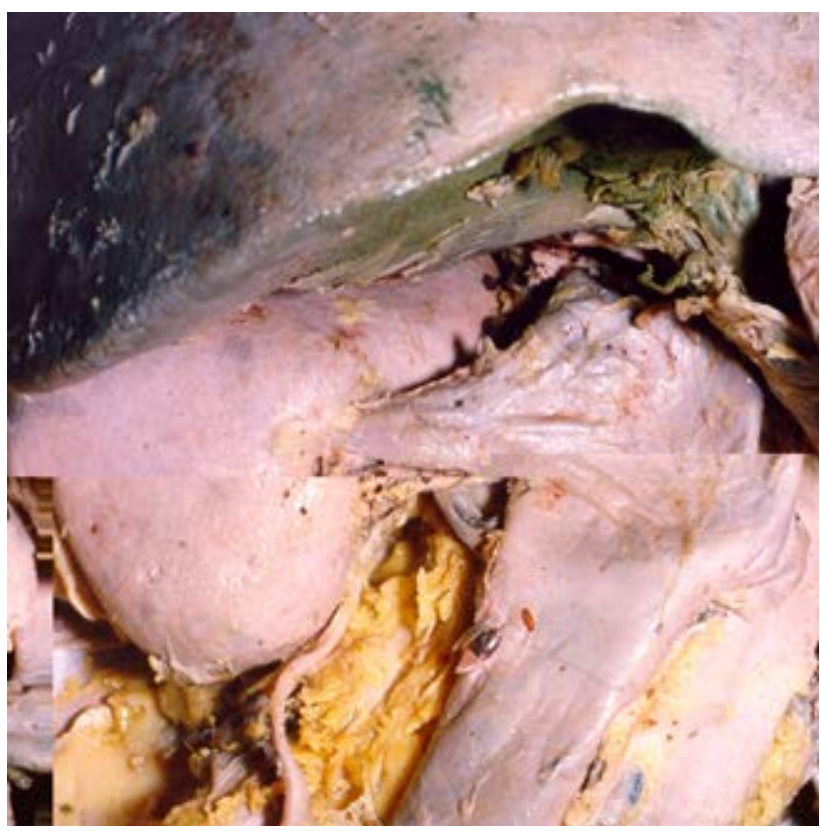

Figure 1 - Right renal vein formed inside the renal sinus. The vein is located anterior to the artery that it covers almost entirely.

The level of formation of the right renal vein was studied on 122 cases, describing the following types: intrasinusal in 24 cases $(19.67 \%$ of cases), juxtarenal in 34 cases $(27.87 \%$ of cases), the most common being the extrarenal in 64 cases $(52.46 \%$ of the cases), with several types:

- closer to the medial border of the kidney, in 22 cases (18.03\% of cases);

- mid distance kidney-inferior vena cava, in 27 
cases $(22.13 \%$ of cases);

- closer to the inferior vena cava in 15 cases $(12.30 \%$ of cases).

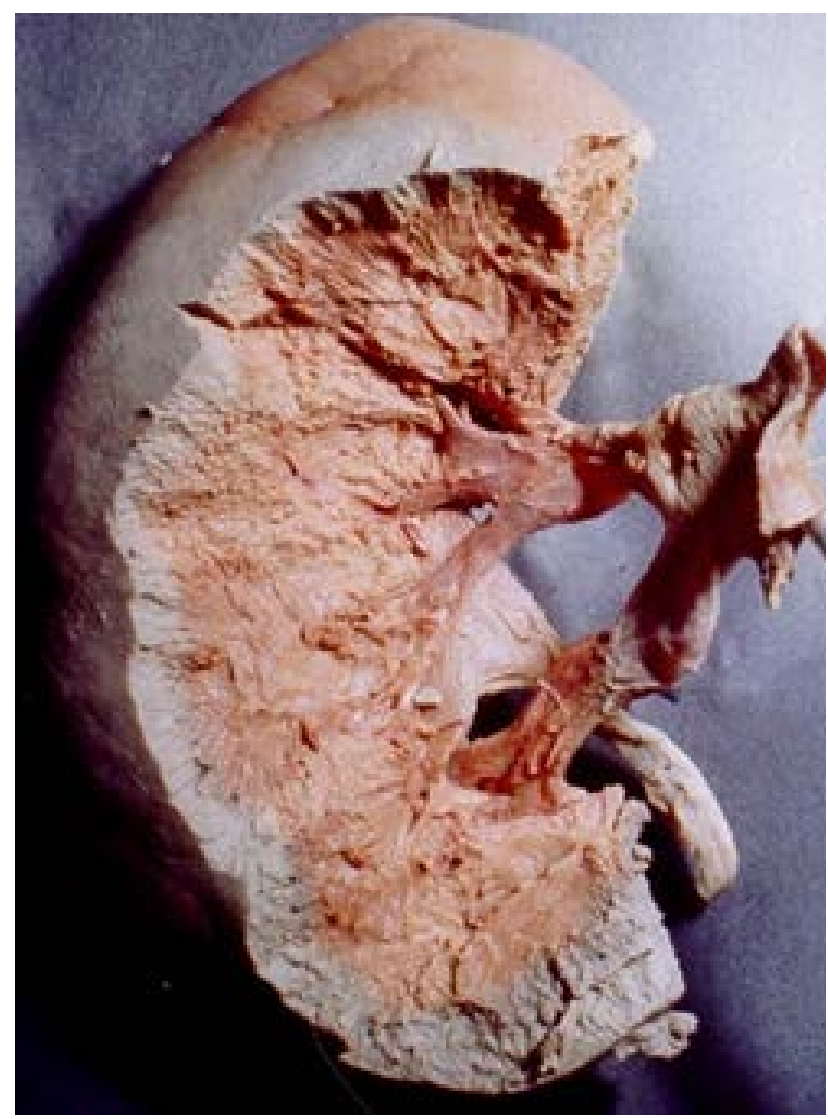

Figure 2-Right renal vein formed outside the hilum, closer to the kidney, from 2 renal trunks: antero-superior, that receives the superior polar vein and inferior polar that receives tributaries from the inferior one third of the kidney.

Between the tributaries of the renal vein frequently appear oblique or vertical anastomoses, sometimes with considerable volume, a detailed description of these anastomoses being made by $[9,10,11,12,13,14] ;[13,14,15,16]$ described in detail the intersegmental anastomoses. Rarely, we met anastomoses between the superior venous trunk of origin of the renal vein and inferior suprarenal vein.

According to $[1,2,3,4,5,6]$ the right renal vein trunk is formed at any level between the renal hilum and the inferior vena cava, and according to $[8,9]$ are formed in the renal hilum. According to
$[7,11,15,16,17]$ they are formed in the renal hilum or beyond.

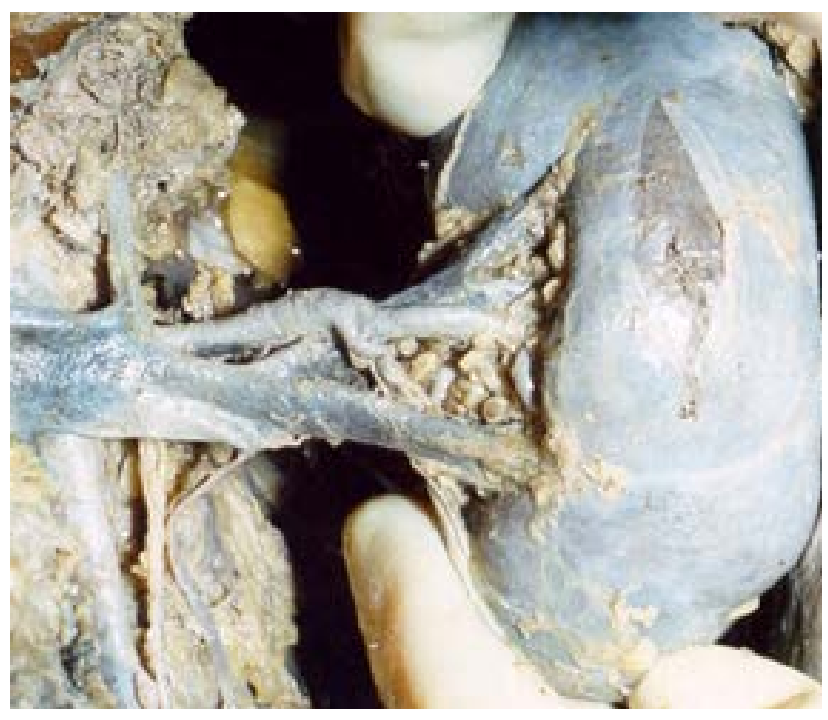

Figure 3-Left renal vein formed outside the hilum, at mid-distance kidney-aorta, from 2 venous trunks: anterosuperior and antero-inferior.

Regarding the formation of the renal veins, we studied a total of 114 cases, finding the following:

- in 64 cases $(56.14 \%$ of cases) the renal vein formed from two venous trunks, either one anterior and the other posterior or both branches are anterior, one superior and one inferior;

- from three venous trunks in 44 cases $(38.60 \%$ of cases);

Both types are cited in the literature $[1,2,3,4,5,6,7,8,18]$, without, however, be stated as a percentage, with the exception of [6].

- in 6 cases $(5.26 \%$ of cases) the renal vein formed from four venous trunks; not did not found cases of renal vein form more than 4 branches, rarely reported cases in literature by $[1,2,3,8,15,16]$, and may reach 5-6 branches [1,2].

Comparing with the results found by [15] and [16], we noticed that these authors find no more than 4 branches that form the renal vein ( $32 \%$ of cases); our percentage was higher with 24 percent for cases with two branches (6 finding $32 \%$ of cases) while the percentage was close for the cases with the formation from three branches (only higher by 2.60 percent, 6 finding $36 \%$ of cases). 


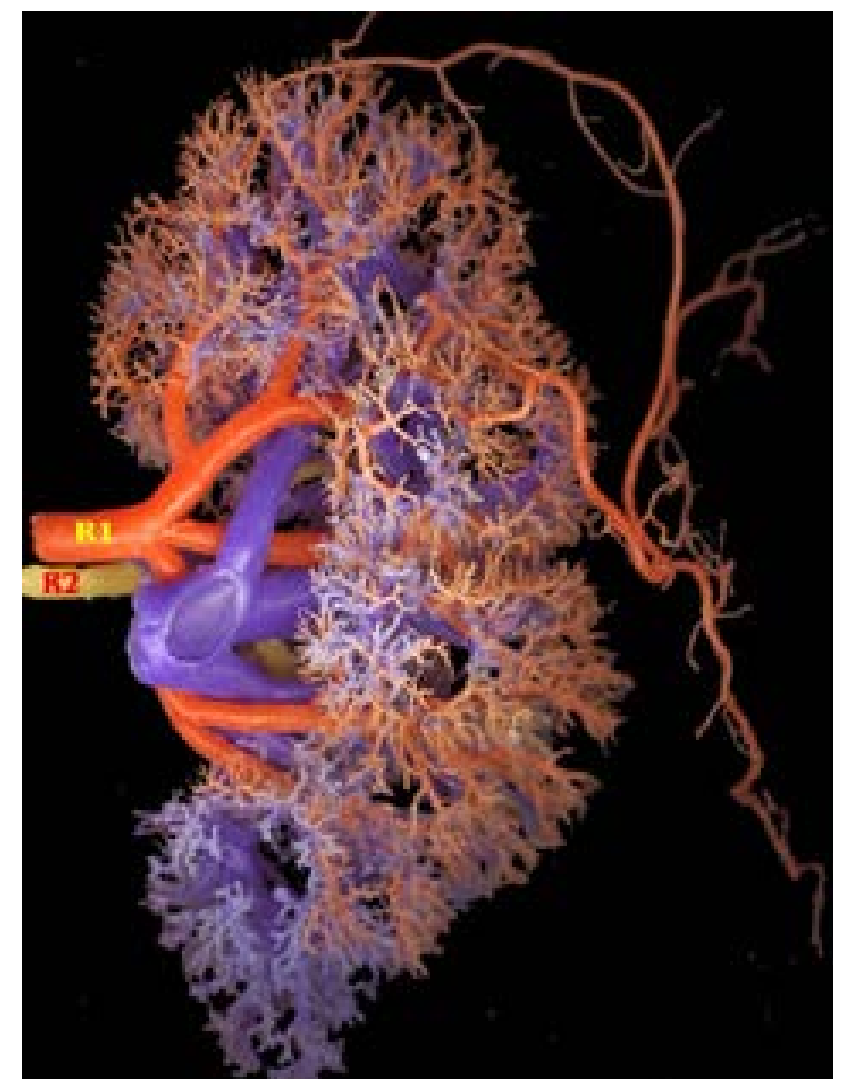

Figure 4-Renal vein formed intrarenal from 3 voluminous venous trunks.

When all trunks are anterior, the veins that originate in the posterior part of the kidney are tributaries to one or more anterior venous branches, on their way to the anterior trunks, the venous branches passing through the arterial branches and through the calyces, an aspect also described by $[1,2,6,7]$.

We noticed the absence of the posterior vein in about 46 cases ( $40.35 \%$ of cases), the end of the venous branches being, most often, into the anterior mesorenal vein and, rarely, into a polar vein (usually in the superior one). Also, frequently, the renal posterior veins may terminate both into the anterior mesorenal and the polar veins, so that virtually all anterior branches receive tributaries from the posterior of the kidney, variants found by $[6,7,19,20]$.

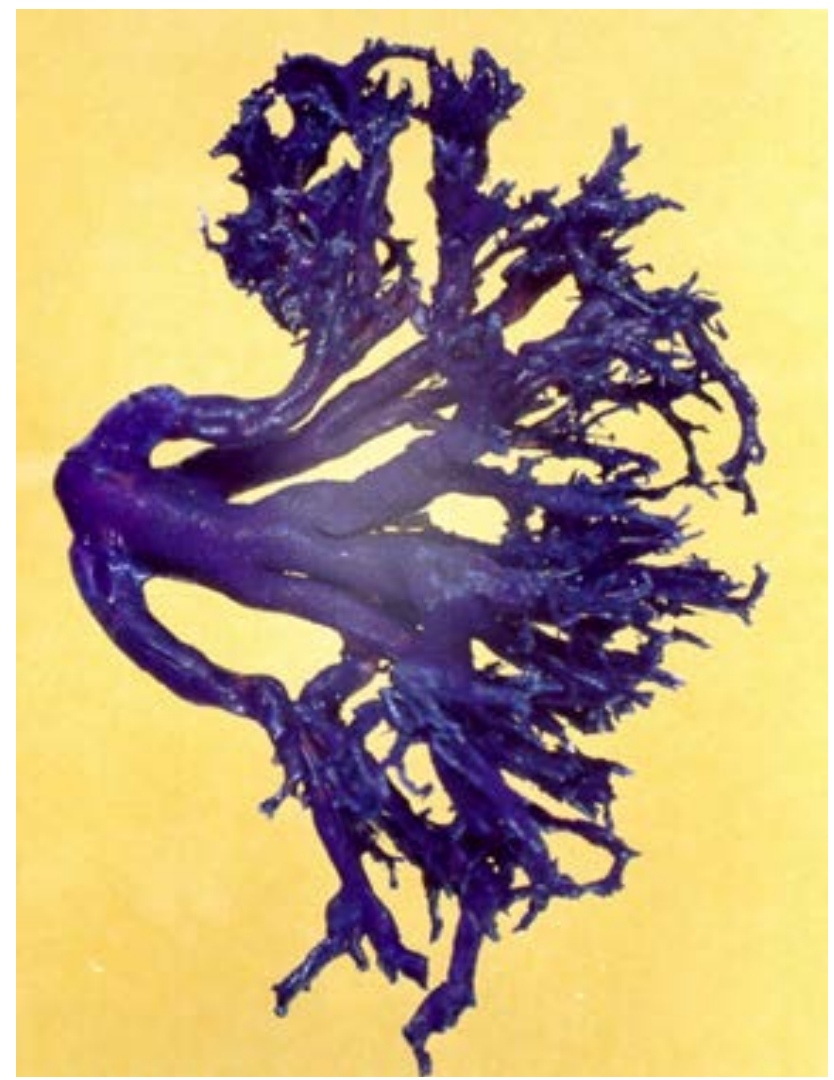

Figure 5-Corrosion cast of a renal vein formed by 4 venous trunks.

Most commonly, the posterior vein is smaller than the anterior and rarely the two veins may be of equal size, an aspect signaled by $[1,3,6,15,16,19,20]$. Sometimes, although present, the posterior vein is very thin, having a caliber smaller than the polar veins or of the branches of the mesorenal one.

The polar renal veins, the superior and the inferior, have a strictly limited territory to their pole or to the corresponding renal third. A polar vein may drain either the posterior face or both faces of the kidney towards the mesorenal vein, to the renal vein or to one of the tributaries of the renal vein. be within the vasculature at which it drains or on the back side, or both sides of the kidney. The latter aspect is more common for the superior polar vein, most often being extrarenal, closer to the kidney.

There are cases that do not form a polar venous trunk and the polar territory renal is drained by thin and multiple veins, tributaries to the anterior mesorenal veins and to the posterior vein. 


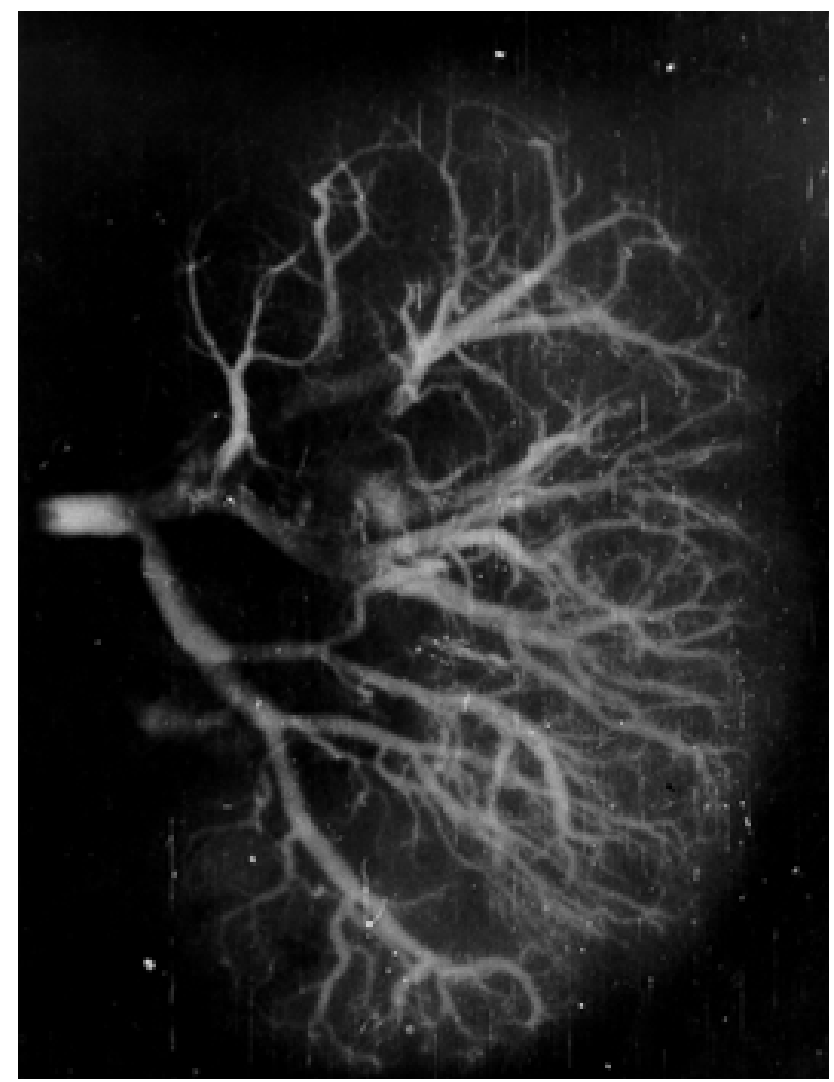

Figure 6-Renal vein formed from two trunks; the posterior vein ends into the antero-superior one. Barium sulphate injection sample.

When the superior polar vein ends into the mesorenal vein at the level of the renal sinus, it goes either between the upper and middle calyces or often on the anterior-medial face of the superior calyx, but almost always closer to the anterior face of the pelvis, as mentioned by $[6,8,20]$.

When there are two polar veins, anterior and posterior, these can be merged into a single venous trunk or may terminate separately, one into the anterior and another into the posterior or both into the anterior.

\section{Conclusions}

Among all levels of the renal veins, the extrarenal formation is most convenient in situations where intervention on the renal pedicle is required and, by analogy with how the renal artery ends (extrarenal), we called this type the ideal, master or maitre-type. The formation of the renal veins, although without many common variants or abnormalities, is of great importance in the overall context of the renal vasculature; it is particularly important in dealing with exorenal vessels and especially the renal vein tributaries which it receives: inferior suprarenal veins on both sides and left genital vein. The pattern and the site of formation of the renal vein directly influence the possibilities for surgical approach to the renal pedicle, a long renal pedicle with the renal artery dividing locorenal and the renal vein formed exorenal facilitating the surgical act when it is necessary.

In the formation of single renal veins particularly strikes the non-correspondence between the venous and the arterial branches. Also, are very frequent the asymmetries between the formation of the two renal veins, with differences from the contralateral vein. The branches of origin of the single renal vein are usually of different sizes and rarely they have the same caliber; the caliber of the mesorenal tributaries is larger than the tributaries to the polar venous branches.

\section{References}

1. Paturet G. (1958). Traité d'anatomie humaine Tome III Fasc. I Appareil circulatoire. (pp. 511526). Paris: Ed. Masson

2. Rouvière H. (1998). Anatomie humaine descriptive, topographique et functionelle. Tome II. Tronc. (pp. 223, 534). Paris: Ed. Masson

3. Testut L. (1921). Traité d'anatomie humaine. Tome deuxième. Angéiologie-Système nerveux 
central. (pp. 213-215). Paris: Ed. Gaston Doin

4. Testut L.\& Latarjet A. (1949). Traité d'anatomie humaine. Tome cinquième. Peritoine. Appareil uro-génital. (pp. 99-151). Paris: Ed. Gaston Doin

5. Juskiewenski S. \& Guitard J. (1994). Les reins et les uretères. In: CHEVREL J.P. - Anatomie clinique. Le Tronc. (pp. 487-502). Paris: Ed. Springer

6. Le Floch-Prigent P. (1987). Biométrie des veines rénales: dissection de 200 sugets frais. Bulletin de l'Association des Anatomistes. 71, 45 - 50

7. Delmas V. (1983). Rein et rachis. Essai sur la segmentation et les variations de l'appareil urinaire a l'etage lombaire. Mémoire DERBH $\mathrm{d}$ 'Anatomie, Bibliothèque anatomique laboratoire d'anatomie, Paris: UER des SaintsPères

8. Gillot C. \& Gallegos A. (1966). Anatomie topographique des veines rénales chez l'homme, Ass. Anat. 135 - 429

9. Poirier P. (1896). Traité d'anatomie humaine. Tome deuxième. (pp. 775-777). Paris

10. Poirier P. \& Charpy A. (1901). Traité d'anatomie humaine. Tome cinquième. (pp. 1-55). Paris

11. Bérard P.H. \& Pouyet M. (1968). Les voies d'évacuation veineuse du rein après ligature de la veine rénale gauche. Extrait du Lyon chirurgical., 64(5), $781-785$
12. Mandarin C. \& Lacerda A. (1933). Veines intrarénales. Étude de l'angioarchitecture segmentaireetdesanastomosesintersegmentoires, Journal d'urologie. 5, 341-344

13. Stolic E. \& Mrvaljevic D. (1971). Les anastomoses intrarenales des veines renales chez l'homme. C. R. Ass. Anat. 146

14. Stolic E. \& Mrvaljevic D. (1967). La topographie intra-rénale des veines rénales chez l'Homme. $C$. R. Ass. Anat. $1106-1111$

15. Anson B. J. \& Daseler E. H. (1961). Common variations in renal anatomy affecting blood supply form and topography. Surg. Gynecol. Obst. 112, $439-449$

16. Anson B. J., Richardson G. A. \& Minear W. L. (1936). Variations in the number end arrangement of the renal vessel: A study of the blood supply of 400 kidneys. J. Urol. 36, $211-219$

17. Gregoire R. (1920). L'appareille uro-génitaI. Paris: Ed. Masson.

18. ***** Gray's Anatomy. (1989). WilliamsWarwick Dysson Bannister. London: Ed. Churchill Livingstone, 218, 248

19. Couinaud C. (1983). Anatomie de l'abdomen. Petit basseri excepte. Tome II Ed. Paris: Ed. G. Doin, 582-596

20. Papin E. \& Jungano (1910). Étude sur la circulation veineuse du rein. Ann. Org. Gén. Ur. $13,1153-1194$ 